\title{
Emulation of Conduction System Functions in the Hearts of Early Mammalian Embryos
}

\author{
THOMAS R. LLOYD AND H. SCOTT BALDWIN \\ University Heart Center, Children's Research Center, and Department of Pediatrics (Cardiology), University of \\ Arizona College of Medicine, Tucson, Arizona 85724 [T.R.L.] and Department of Pediatrics (Cardiology), \\ University of Pennsylvania College of Medicine, Philadelphia, Pennsylvania 19104 [H.S.B.]
}

\begin{abstract}
The conduction system functions of atrioventricular sequential contractions, atrioventricular delay, and coordination of ventricular contraction were examined in rat embryos at the earliest functional stage of cardiac development (before cardiac looping, $n=6$ ) and shortly after looping $(n=15)$. Atrioventricular sequential contractions were observed in all embryos, and contractions appeared to originate in the left sinus horn. Atrioventricular delay was present in both prelooped $(132 \pm 32 \mathrm{~ms})$ and looped $(141 \pm 15 \mathrm{~ms})$ hearts. Before looping, contractions traveled from proximal ventricle to bulbus cordis, a distance of $253 \pm 27 \mu \mathrm{m}$, in $72 \pm 22 \mathrm{~ms}$. After looping, contractions crossed an increased intraventricular distance $(520 \pm 28 \mu \mathrm{m}, p<0.005)$ in substantially less time $(16 \pm$ $7 \mathrm{~ms}, p<0.005)$. Sinoatrial and atrioventricular nodal functions are emulated in both prelooped and looped hearts of early mammalian embryos, and His-Purkinje system function is emulated in looped hearts. (Pediatr Res 28: 425-428, 1990)
\end{abstract}

In the mature heart, the specialized conduction system serves to provide an appropriate heart rate, atrioventricular sequential contraction with appropriate atrioventricular delay, and coordinated ventricular contraction. The early embryonic heart, which has not developed a sinoatrial node, atrioventricular node, or His-Purkinje system, must nonetheless provide effective circulation if the embryo is to remain viable, and the embryonic heart depends strongly on appropriate heart rate and atrioventricular sequential contraction to maintain optimal cardiac output $(1,2)$. In the avian embryo, pacemaker function is served by the left sinus horn, and the myocardium of the atrioventricular canal provides atrioventricular conduction with appropriate delay before development of the sinoatrial and atrioventricular nodes (3, 4). We studied rat embryos in the earliest functional stages of heart development to examine development of atrioventricular sequential contraction, atrioventricular delay, and coordination of ventricular contraction in a mammalian system. We studied the mechanical, rather than electrical, manifestations of these phenomena because electrophysiologic development sometimes precedes development of the mechanical events (3) and because it is the mechanical events that are essential for effective function of the embryonic heart.

\section{MATERIALS AND METHODS}

Embryo culture. Whole embryo culture was performed as previously described (5). Wistar rat embryos were explanted on

Received March 29, 1990; accepted June 26, 1990.

Correspondence: Thomas R. Lloyd, M.D., Pediatric Cardiology, Arizona Health Sciences Center, Tucson, AZ 85724

Supported by NIH awards HL02197, HL67413, HL43600, and HD18577. T.R.L. and H.S.B. were also supported by NRSA HL07413. gestational d 9.5, d 0 being the day of positive vaginal smear. Embryos were explanted in Tyrode's solution, the decidual mass was removed, and Reichert's membrane was opened. Embryos were then placed in roller bottles containing heat-inactivated rat serum and cultured under an atmosphere of $90 \% \mathrm{~N}_{2}, 5 \% \mathrm{O}_{2}$, and $5 \% \mathrm{CO}_{2}$ as described by New (6). At explanation, embryos contain up to three somites and the primitive heart tube has not yet formed. Embryos were studied either at the five- to sevensomite stage ( $24 \mathrm{~h}$ in culture), at which stage the heart tube has formed but has not begun to loop, or at the 13- to 15-somite stage ( $36 \mathrm{~h}$ in culture), after cardiac looping is complete but before development of the atrial or ventricular septa.

Wall motion recording. Embryos with prelooped or looped hearts were examined in Tyrode's solution using a Wild M5 microscope. Embryos were positioned and immobilized as shown in Figure 1 in a depression made in 9\% agar. Embryos were maintained at $37.5 \pm 0.5^{\circ} \mathrm{C}$ by a thermostatically-controlled stage warming/cooling device (Sensortek, Clifton, NJ). Video-tape recordings of the embryonic hearts (at $100 \times$ and $200 \times$ magnification) were made using high-resolution closed circuit video and electronic processing to produce monochrome images of maximum contrast. For wall motion recording, these videotape recordings were played back on a 19-inch monitor. Fiber optic cables were placed over the video images of the proximal atrium, distal atrium, proximal ventricle, and proximal bulbus cordis as indicated in Figure 1. The fiber optic cables were connected to photocell amplifiers that converted the changes in light intensity of the video image caused by wall motion to voltage signals, which were then recorded by a multichannel recorder at a paper speed of $50 \mathrm{~mm} / \mathrm{s}$. This apparatus has been described previously (7). Because of the nature of the optical system, the moment of peak wall motion is the most reliably detected point. Peak wall motion is also less affected by motion of adjacent structures than earliest motion would be, so position of the fiber optic cables was adjusted to optimize recording of peak wall motion. Cycle length was measured from the most proximal site recorded. Intervals between peak wall motion in the proximal and distal atrium (intra-atrial delay), distal atrium and proximal ventricle (atrioventricular delay), and proximal ventricle and proximal bulbus cordis (intraventricular delay) were measured to the nearest $5 \mathrm{~ms}$ as shown in Figure 2. Each measurement reported was the mean of five sequential contractions. Linear distance between recording sites was measured on the video screen and converted to $\mu \mathrm{m}$ by reference to micrometer calibration recorded on each videotape at the same magnifications.

Statistical analysis. Intervals between maximum contraction within the atrium and ventricle and atrioventricular delay in prelooped and looped hearts were analyzed by analysis of variance. The relationship between cycle length and atrioventricular delay was examined by linear regression analysis. The Scheffe method was used to correct for multiple comparisons, and $p<$ 0.05 was chosen to indicate statistically significant differences. 

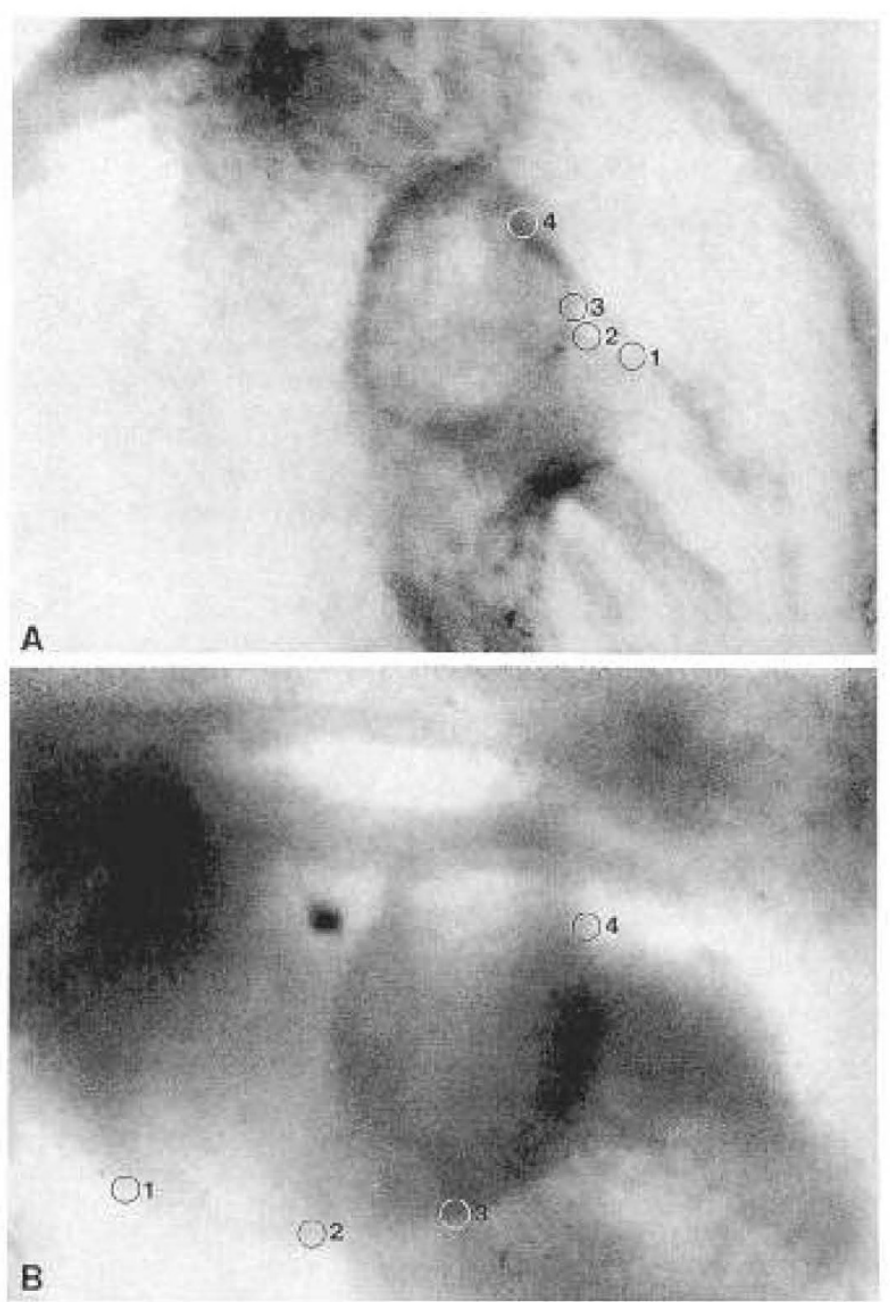

Fig. 1. Still images from video recordings of rat embryos with prelooped $(A)$ and looped $(B)$ hearts. Both embryos are oriented with the ventricle and bulbus cordis vertical; in $A$, the anterior neural fold is visible at the top, whereas in $B$, it is partially seen at the lower right. The circles represent sites of optical wall motion recording: 1 , proximal atrium; 2, distal atrium; 3, proximal ventricle; and 4, proximal bulbus cordis. Intra-atrial distance is measured from $I$ to 2 , and intraventricular distance is measured from 3 to 4 .

\section{RESULTS}

Data obtained from 15 embryos with looped hearts and six embryos with prelooped hearts are shown in Table 1 . Contrast was not sufficient to produce adequate tracings of wall motion at all sites in some embryos, but atrioventricular sequential contraction was evident in all embryos. Atrial contraction appeared to be of low amplitude in prelooped hearts, and contraction passed from the left sinus horn to the atrioventricular canal in a visible wave. This mode of contraction, which we have termed peristaltic, was also observed in the atria of the looped hearts, but because of their orientation it was not possible to distinguish the exact site of earliest contraction in looped hearts. Ventricular contraction also appeared peristaltic in prelooped hearts, but in looped hearts all portions of the ventricle appeared to contract more or less simultaneously, with mean intraventricular delay of $16 \mathrm{~ms}$, which is at the frequency response limit of the video system $(60 \mathrm{~Hz})$. As shown in Table 1 , the delay in peak contraction between proximal ventricle and proximal bulbus cordis decreased with looping while the distance between ventricle and bulbus cordis increased, resulting in a velocity of apparent propagation of ventricular contraction (distance $\div$ delay) nearly $10 \times$ higher in looped than in prelooped hearts. There were no significant differences between intra-atrial delay in looped hearts
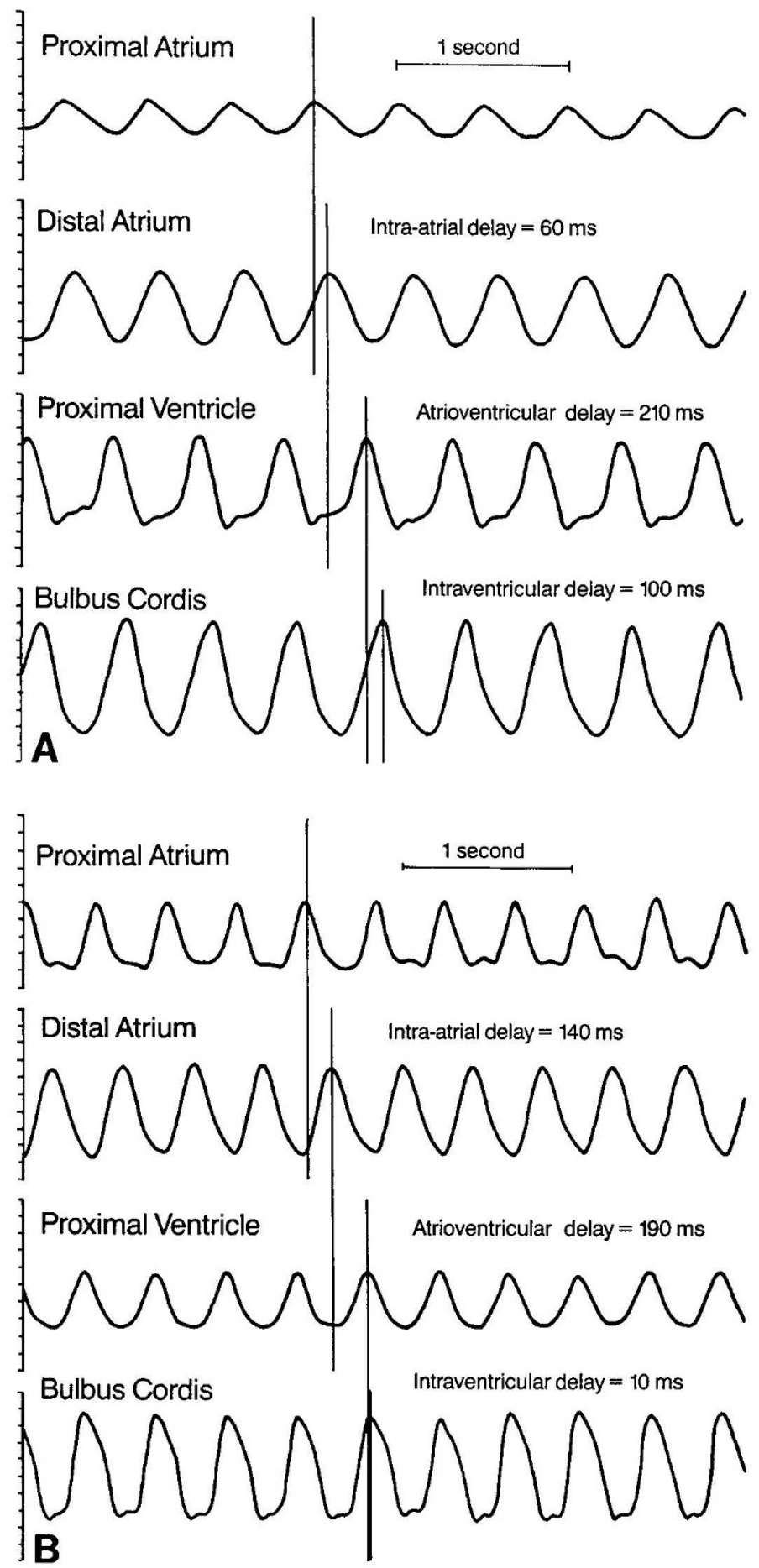

Fig. 2. Optical wall motion recordings from the embryos shown in Figure 1. $A$, prelooped heart; $B$, looped heart. The vertical lines show measurement of intra-atrial delay from peak wall motion in the proximal atrium to peak wall motion in the distal atrium, atrioventricular delay from peak wall motion in the distal atrium to peak wall motion in the proximal ventricle, and intraventricular delay from peak wall motion in the proximal ventricle to peak wall motion in the bulbus cordis.

and intraventricular delay in prelooped hearts. Contractile motion of the proximal atria in prelooped hearts was generally very faint, preventing measurement of intra-atrial delay in all but one specimen. Intra-atrial delay $(98 \mathrm{~ms})$ in this embryo, which appeared fairly typical, was similar to intra-atrial delay in prelooped hearts and intraventricular delay in looped hearts.

Atrioventricular delay did not differ significantly between looped and prelooped hearts. A weak positive correlation was 
Table 1. Emulation of conduction system function: measurements in early mammalian embryos (all data mean $\pm S E M$ )

\begin{tabular}{lcc}
\hline \multicolumn{1}{c}{ Measurement } & Prelooped hearts & Looped hearts \\
\hline Intra-atrial delay $(\mathrm{ms})$ & 98 & $80 \pm 15$ \\
& $n=1$ & $n=11$ \\
Intra-atrial distance $(\mu \mathrm{m})$ & 163 & $287 \pm 46$ \\
& $n=1$ & $n=11$ \\
Atrioventricular delay $(\mathrm{ms})$ & $132 \pm 32$ & $141 \pm 15$ \\
& $n=4$ & $n=14$ \\
Intraventricular delay $(\mathrm{ms})$ & $72 \pm 22$ & $16 \pm 7^{*}$ \\
& $n=5$ & $n=14$ \\
Intraventricular distance $(\mu \mathrm{m})$ & $253 \pm 27$ & $520 \pm 28^{*}$ \\
& $n=5$ & $n=14$ \\
Cycle length $(\mathrm{ms})$ & $558 \pm 32$ & $448 \pm 41$ \\
& $n=6$ & $n=15$ \\
\hline
\end{tabular}

${ }^{*} p<0.005$ vs prelooped.

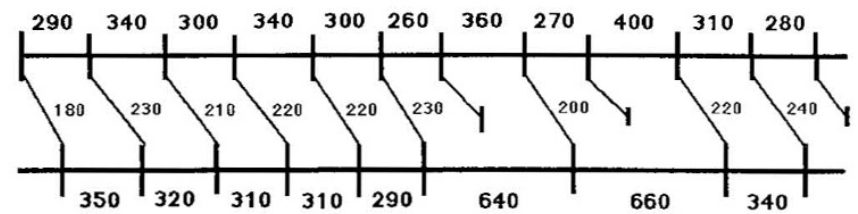

Proximal ventricle

A.

\section{Distal atrium}

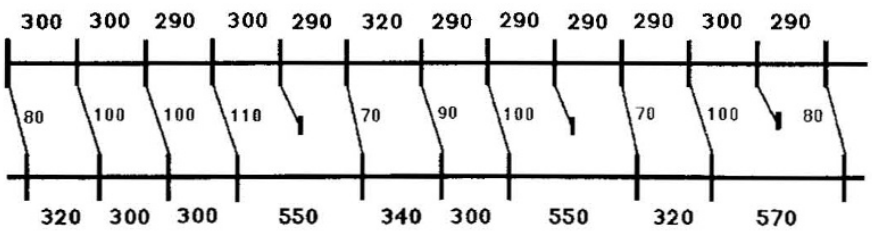
Proximal ventricle

B.

Fig. 3. Ladder diagrams of optical wall motion recordings from dista atrium and proximal ventricle showing atrioventricular Wenckebach periodicity in embryos with prelooped $(A)$ and looped $(B)$ hearts.

found between cycle length and atrioventricular delay that was not statistically significant $(r=0.261, p=0.30)$. Atrioventricular Wenckebach periodicity was transiently observed in five looped and three prelooped hearts, as shown in Figure 3.

\section{DISCUSSION}

Our observations demonstrate atrioventricular sequential contraction in the early mammalian embryo before development of the atrioventricular node. Similar findings have been reported in avian embryos (4). Just as the left sinus horn emulates the function of the sinoatrial node as the dominant pacemaker of the early embryonic heart (3), the myocardium of the atrioventricular canal emulates the function of the atrioventricular node by allowing atrioventricular sequential contraction and providing an appropriate atrioventricular delay. In the avian embryo, the myocardium of the atrioventrice ${ }^{\circ}$ canal has been shown to share cell action potential chara : ristics with atrioventricular nodal cells (8), and our study in má malian embryos has shown that the myocardium of the atrioventricular canal shares with the atrioventricular node the functional properties of atrioventricular delay and potential for Wenckebach periodicity. We believe that the Wenckebach periodicity we observed is not a normal part of cardiac development, but rather reflects some instability in our preparations. Of interest, Wenckebach periodicity was most often observed with stage temperatures $\geq 38^{\circ} \mathrm{C}$. In addition to its unique (in the early embryo) electrophysiologic properties, the myocardium of the atrioventricular canal differs from atrial or ventricular myocardium in its ability to induce endocardial cushion formation (9) and in its ultimate fate, which is to be replaced by the fibrous tissue of the atrioventricular ring. It is tempting to speculate that accessory atrioventricular connections, which more often exhibit electrophysiologic characteristics of working myocardium than of atrioventricular node $(10$ 11), have their origin in strands of atrioventricular canal myocardium that fail to electrophysiologically differentiate from adjacent myocardium and that subsequently fail to yield their position to fibrous connective tissue.

In adult mammals and birds, the His-Purkinje system allows synchronization of ventricular contractions. We have observed that the mammalian ventricle in its earliest functional stage, i.e. before cardiac looping, exhibits contractions that move in a wave from the atrioventricular canal to the conus arteriosus. After looping, however, ventricular contraction is much more synchronous, emulating function of the His-Purkinje system. It is, of course, incumbent on the embryonic heart to have mechanisms such as this that shorten the duration of mechanical systole because cycle length also shortens during development (12). Because we examined mechanical rather than electrical events, we cannot determine whether this effect resulted from more rapid electrical conduction or from differential changes in electromechanical coupling. Although controversy remains as to whether the bundle branches and distal Purkinje cells arise in situ in the ventricles or whether the bundle of His extends from its origin in the septum primum to produce the Purkinje network (13-15), there is no evidence of Purkinke cell precursors in the ventricle at stages as early as were examined in this study. Synchronous contraction should be more effective than more peristaltic contraction, but only if there is atrioventricular valve competence. The endocardial cushions act as an atrioventricular valve in the avian embryo heart $(16,17)$; we speculate that the change from peristaltic to synchronous ventricular contraction may not be favorable until the atrioventricular valve-like function of the endocardial cushions is well established.

Conclusions. We have shown that conduction system functions are emulated in the hearts of mammalian embryos at their earliest functional stages. Earliest pacemaker function is seen in the left sinus horn and atrioventricular delay is produced at the atrioventricular canal, similar to observations in avian embryos. Emulation of His-Purkinke system function was demonstrated in postlooped hearts, a previously unreported phenomenon. The mechanisms by which these embryonic conduction system functions and their mature counterparts develop and the possible relationship between the Wolff-Parkinson-White syndrome and the unique developmental characteristics of the atrioventricular canal myocardium are important areas for further study.

\section{REFERENCES}

1. Dunnigan A, Hu N, Benson Jr DW, Clark EB 1987 Effect of heart rate increase on dorsal aortic flow in the stage 24 chick embryo. Pediatr Res 22:442-444

2. Cuneo B, Hughes S, Benson Jr DW 1989 Heart rate change in the stage 24 chick embryo: effect on stroke volume. Am J Cardiol 64:413(abstr)

3. Kamino K, Komuro H, Sakai T, Hirota A 1988 Functional pacemaking area in the early embryonic chick heart assessed by simultaneous multiple-site optical recording of spontaneous action potentials. J Gen Physiol 91:57359

4. Argüello C, Alanis J, Pantoha O, Valenzuela B 1986 Electrophysiologic and ultrastructural study of the atrioventricular canal during the development of the chick embryo. J Mol Cell Cardiol 18:499-510

5. Baldwin HS, Solursh M 1989 Hydration of the cardiac extracellular matrix is not required for looping of the mammalian heart in situ. Dev Biol 136:555559

6. New DAT 1987 Whole-embryo culture and the study of mammalian embryos during organogenesis. Biol Rev Cambridge Philosophic Soc 53:81-122

7. Marvin WJ, Chittick VL, Rosenthal JK, Sandra A, Atkins DL, Hermsmeyer K 1984 The isolated sinoatrial node cell in primary culture from the newborn rat. Circ Res 55:253-260

8. Argüello C, Alanis J, Valenzuela B 1988 The early development of the atrioventricular node and bundle of His in the embryonic chick heart. An electrophysiological and morphological study. Development 102:623-637

9. Markwald RR, Runyan RB, Kitten GT, Funderburg FM, Bernanke DH, 
Brauer PR 1984 Use of collagen gel cultures to study heart development: proteoglycan and glycoprotein interactions during the formation of endocardial cushion tissue. In: Trelstad RL (ed) The Role of Extracellular Matrix in Development. Alan R Liss, New York, pp 323-350

10. Inoue H, Zipes DP 1987 Conduction over an isthmus of atrial myocardium in vivo: a possible model of Wolff-Parkinson-White syndrome. Circulation 76:637-647

11. Klein GJ, Guiraudon GM, Kerr CR, Sharma AD, Yee R, Szabo T, Yeung Lai Wah JA 1988 "Nodoventricular" accessory pathway: evidence for a distinct accessory atrioventricular pathway with atrioventricular node-like properties. J Am Coll Cardiol 11:1035-1040

12. Robkin MA, Shepard TM, Tanimura T $1972 \mathrm{~A}$ new in vitro culture technique for rat embryos. Teratology 5:367-376
13. Canale E, Smolich JJ, Campbell GR 1987 Differentiation and innervation of the atrioventricular bundle and ventricular Purkinje system in sheep heart. Development 100:641-651

14. de Groot IJM, Sanders E, Visser SD, Lamers WH, de Jong F, Los JA, Moorman AFM 1987 Isomyosin expression in developing chicken atria: a marker for the development of conductive tissue? Anat Embryol (Berl) 176:515-523

15. Vassall-Adams PR 1982 The development of the atrioventricular bundle and its branches in the avian heart. J Anat 134:169-183

16. Van Mierop LHS, Bertuch CJ 1971 Development of arterial blood pressure in the chick embryo. Am J Physiol 212:43-48

17. Ruckman RN, Cassling RJ, Clark EB, Rosenquist GC 1981 Cardiac function in the embryonic chick. In: Pexieder T (ed) Perspectives in Cardiovascular Research. Raven Press, New York, pp 401-417

\section{Announcement}

\section{Joint PhD-Fellowship Training Program}

The University of Chicago Department of Pediatrics announces its unique, new Pediatric Science Training/ $\mathrm{PhD}$ Program. Trainees wishing to pursue an academic career may simultaneously pursue subspecialty training and a graduate school program leading to the $\mathrm{PhD}$ degree in this 5-year program. Training is available in most pediatric subspecialties, and research opportunities are available in diverse fields, including the social and behavioral sciences as well as the biologic sciences. Applications are being accepted for 1991. This program is funded by the National Institutes of Child Health and Human Development. Consequently, the program is only open to U.S. citizens or permanent residents. Address inquiries to: Robert L. Rosenfield, M.D., Program Director, Pediatric Science Training/PhD Program, Wyler Children's Hospital, 5841 South Maryland, Chicago, IL 60637. 